\title{
Checkpoint Inhibitors in Breast Cancer - Current Status and Future Directions
}

\author{
Joachim Bischoff \\ Center for Clinical Trials, Städtisches Klinikum Dessau, Dessau, Germany
}

\author{
Keywords \\ Triple-negative breast cancer - Immune oncology . \\ PD-1/PD-L1 system - Expression · Checkpoint inhibitors . \\ Monoclonal antibodies
}

\section{Summary}

Antineoplastic agents directly targeting tumor cells have represented the major strategy of systemic anticancer therapy for many years. Nevertheless, overcoming resistance mechanisms remains a great challenge because treatment options are limited in many cases. From this point of view, immunotherapeutic approaches seem promising in a broad spectrum of solid tumors. These include in particular the currently available inhibitors directed against immune checkpoints leading to a significant T-cell activation. To date, the programmed death receptor 1 (PD-1) and its ligand are the most prominent targets in this context. However, the role of checkpoint inhibitors in the treatment of breast cancer is still being debated, and the main focus is on triple-negative breast cancer patients as a target population in many ongoing trials. Moreover, the potential superiority of combinations with other anticancer drugs such as cytotoxics and targeted agents will be discussed.

(C) 2018 S. Karger GmbH, Freiburg

\section{Introduction}

In the last decade, scientific research in the field of immune oncology (IO) was dominated by the characterization of immune checkpoints in the context of T-cell activation. These checkpoint receptors expressed on immune cells inhibit the function of $\mathrm{T}$ cells leading to avoidance of autoimmunity. However, these mechanisms are also being hijacked by tumors to evade immune attack. The recent renaissance of cancer immunotherapy has been largely attributed to the development of novel immunotherapeutic agents which target-specific immunoregulatory checkpoints to enhance the endogenous antitumor immune responses.

In the early 1990s, a growing number of functionally related molecules (B7:CD28 family and tumor necrosis factor receptor (TNF-R) family) were described that ultimately influence the amplitude and quality of the immune response via the balance between co-stimulatory and co-inhibitory signals at the immune synapse (immune checkpoint molecules) [1]. Different kinetics of immune checkpoint molecules (e.g., CD28 is expressed on naive and resting T cells, whereas CTLA-4 is upregulated upon T-cell activation) or contextdependent signaling of one receptor, e.g., the cytoplasmic tail of programmed death receptor 1 (PD-1) containing an immune-tyrosine switching motif, can mediate either positive or negative signals [2, 3]. This complexity of immune checkpoint signals is further increased since these molecules tune various steps of an immune response and thus the active immune cell repertoire including the thymic T-cell selection, initial activation and proliferation in secondary lymphoid organs, T-cell trafficking to the peripheral sites, effector and helper functions, and the development of memory $\mathrm{T}$ cells [4]. In addition, immunosuppressive cells, like regulatory $\mathrm{T}$ cells (Tregs), tumor-associated macrophages (TAM), and myeloidderived suppressor cells (MDSC), also express immune checkpoint ligands and receptors and therefore could be altered in their own function and/or provide co-stimulatory/co-inhibitory signals to other immune cells via immune checkpoint molecules [5].

Meanwhile, a number of monoclonal antibodies (mAb) directed against several inhibitory checkpoints, such as CTLA4, PD-1, LAG3, TIM3 and OX40, all of them being members of the B7 or TNF-R family, have been developed. Based on encouraging data of a metastatic melanoma phase III trial, the first checkpoint inhibitor (CPI), ipilimumab, was approved in 2010 [6]. Thereafter, several

\section{KARGER}

() 2018 S. Karger GmbH, Freiburg

Fax +497614520714 
second-generation inhibitors were licensed for the treatment of a broad spectrum of solid tumors. These drugs are directed against the PD-1 receptor (nivolumab and pembrolizumab) or its ligand (atezolizumab and durvalumab). The role of this promising IO approach in breast cancer (BC) has yet to be defined, and, so far, only a small number of published trials for this indication exist. Nevertheless, there is some evidence that triple-negative breast cancer (TNBC) is a good candidate for further investigation of the role of $\mathrm{CPI}$ in the future.

\section{PD-1/PD-L1 as a Therapeutic Target}

PD-1 (CD279) was initially identified in a murine T-cell hybridoma undergoing activation-induced cell death [7]. Cross-linking of PD-1 by either one of its ligands, PD-L1 (B7-H1, CD274) or PD-L2 (B7-DC, CD273), results in decreased proliferation, cytokine production, and impaired lytic function of $\mathrm{T}$ cells. So far, there is no convincing data showing that PD-1 signaling directly mediates cell death, indicating that the nomenclature for this receptor and its ligands is misleading. Analogous to CTLA-4, PD-1 is expressed on activated CD4+ T cells, CD8+ T cells, and Tregs, but also on activated B cells and natural killer cells. Thus, CTLA-4 signaling alters the early phase of activation of naive or memory $\mathrm{T}$ cells, while PD-1 signals limit the effector phase of T cells. PD-L2 expression was initially thought to be more restricted to macrophages and dendritic cells (DC) and induced by Th2 (interleukin-4) cytokines. However, recent data indicate that PD-L2 can also be induced on a wide variety of immune and non-immune cells. Next to the classical PD-L1/PD-1 interaction, PD-L1 can also interact with B7-1 expressed on T cells and professional antigenpresenting cells (APC) delivering an additional inhibitory signal [8]. Finally, PD-L1 expression on tumor cells resulted in impaired proliferation arguing for a possible retrograde signal mediated via engagement of PD-L1 by PD-1.

Synchronous PD-L1 and PD-1 expression on T cells suggests that T-to-T-cell interactions also modulate T-cell activation, but this hypothesis has not yet been proven. Chronic antigen exposure, as observed during chronic viral infections or cancer, leads to high PD-1 expression and subsequently induces a state of exhaustion among the antigen-specific $\mathrm{T}$ cells. Indeed, a large proportion of tumor-infiltrating lymphocytes (TILs) from many different tumor types expresses high levels of PD-1 [9]. Two opposing ideas have been postulated: i) Expression of PD-L1 as a result of tumor progression leading to intrinsic immune resistance and immune escape; ii) Since interferon (IFN) $-\gamma$ is known as a primary inducer of PD-L1 expression, TILs may actually trigger their own inhibition by secreting cytokines that drive tumor PD-L1 expression. Thus, adaptive immune resistance via PD-L1 reflects the tumor's adaptation to endogenous immune responses capturing the natural physiology of the PD-1/PD-L1 pathway for tissue protection. Tumor cells take advantage of these mechanisms to evade immune recognition. Therefore, it is not surprising that the interaction between this receptor and its ligand plays a central role in oncology.
This appears to be of clinical relevance also in BC. Recently, it was shown that PD-L1 expression is detectable in a significant number of BC samples - evaluated on either tumor cells or TILs [10]. Furthermore, TNBCs express this protein more frequently than other BC subtypes [11]. Thus, it is discussed whether PD-L1 expression is predictive for the response to the anti-PD-1 mAb in this disease, as recently shown for non-small cell lung cancer (NSCLC). Pembrolizumab was licensed in this indication only for PD-L1-positive patients in the first-line setting. Based on such considerations, a small cohort of heavily pretreated TNBC patients was included into several proof-of-concept trials $[12,13]$.

Results from 2 studies assessing the anti-PD-1 mAb pembrolizumab and the anti-PD-L1 antagonist atezolizumab have since been presented. The pembrolizumab phase Ib KEYNOTE-012 trial recruited 32 metastatic TNBC patients, most of whom had previously received at least 3 lines of chemotherapy for metastatic disease [12]. Only patients with PD-L1 staining in the stroma or in 1\% of tumor cells as evaluated by immunohistochemistry in archived samples were eligible. An extremely promising overall response rate (ORR) of $19 \%$ was described, including 1 complete and 4 partial responders. The atezolizumab phase Ia expansion trial enrolled 54 TNBC patients [13]. Again, these patients were heavily pretreated ( $85 \%$ had received 4 or more lines of chemotherapy). In the 21 TNBC patients in whom efficacy was evaluable, a similar ORR rate of $24 \%$ was reported, including 3 partial and 2 complete responses. In this trial, tumors were considered PD-L1-positive when PD-L1 was expressed in $\geq 5 \%$ of the infiltrating immune cells. The clinical activity of pembrolizumab in this subset has been confirmed by the analysis of an extended cohort of BC patients ( $\mathrm{n}=$ 170) with an ORR of $23 \%$ in the first-line setting [14]. However, it is noteworthy that the efficacy of PD-(L)1 inhibitors in $\mathrm{BC}$ as a single agent appears to be modest.

\section{Chemotherapy and the Antitumor Immune Response}

Numerous reports have highlighted the complex interactions that occur between the immune response and cytotoxic drugs used to treat cancer patients. Both players have the ability to potentially enhance or harm their counterpart and thereby detrimentally affect their antitumor functions. There is accumulating evidence that some cytotoxic drugs, such as anthracyclines, actually promote antitumor immunity and thereby contribute to the therapeutic effect. This is mainly linked to the ability of these drugs to provoke 'immunogenic' cell death (ICD) with the apoptotic cancer cells emitting signals that trigger antitumor immunity [15]. The concept that cell death is either tolerogenic, immunologically silent, or even suppressive has been discussed for several years [16]. The hypothesis of a new immune-stimulating form of cell death is quickly gaining ground, stressing the importance of the way cell death is induced. Initially, ICD was only observed with certain chemotherapeutic agents such as doxorubicin and oxaliplatin [17]. Irradiation was also quickly profiled as a potent mediator [18]. The concept of ICD is based on the release of damage-associ- 
ated molecular patterns (DAMP) [19]. Different DAMP have distinct roles, although they are all involved in eliciting a long-term immune response. High-mobility group box proteins (HMGB) like HMGB1 and heat shock proteins (such as HSP 70) act as proinflammatory signals, adenosine triphosphate (ATP) as a 'find me' signal, and calreticulin as an 'eat me' signal for APC. Unlike freely released ATP, calreticulin and HSP 70 are released as membrane blebs. The release of these danger signals leads to antigen engulfment and the stimulation of Toll-like receptors on DC. ICD facilitates tumor antigen presentation and enables an efficient activation of CD4+ and CD8+ T cells. For this reason, it is assumed that ICD is one of the most important contributors to long-term antitumor responses [20]. These observations support the hypothesis that combinations of cytotoxics and immunotherapeutics are able to induce synergistic antitumor effects. However, so far, only a few preliminary data are available focusing on the neoadjuvant setting in TNBC.

\section{Future Perspectives}

\section{Combination of Immunotherapy with Antiangiogenic Therapy}

When combining targeted agents with immunotherapy, antiangiogenic agents are of particular interest. Angiogenesis is the growth of new microvessels from the preexisting vasculature, a process that involves a fine balance between proangiogenic and antiangiogenic factors and a well-orchestrated coordination between multiple cell types such as macrophages, endothelial cells, and pericytes [21]. Supported by an abnormal tumor microenvironment with interstitial hypertension, hypoxia, and acidosis, both tumor and stromal cells contribute to a shift of this balance towards tumor angiogenesis leading to the formation of new vasculature that is necessary for cancer cells to proliferate beyond microscopic size and to metastasize. The vascular endothelial growth factor (VEGF) plays a key role in the regulation of endothelial cell migration and proliferation necessary for angiogenesis under physiologic conditions and pathophysiologic conditions such as cancer. In addition, VEGF also manifests potent immunoregulatory activity like the inhibition of the differentiation and maturation of DC from hematopoietic progenitors. In mouse tumor models, treatment of established tumors with the anti-VEGF antibody bevacizumab did not affect the rate of tumor growth but significantly improved the number and function of host DC [22]. The administration of a VEGF antibody combined with adoptive T-cell transfer inhibited the growth of established melanoma, enhanced infiltration of $\mathrm{T}$ cells, and prolonged patient survival compared with either monotherapy.

In a phase I study, 46 patients with metastatic melanoma were treated in 4 dosing cohorts with the combination of ipilimumab and bevacizumab. With an ORR and disease control rate (DCR) of 19.6 and $67.4 \%$, respectively, the median survival was 25.1 months. Moreover, bevacizumab and ipilimumab treatment resulted in changes in the tumor vasculature and immune responses. However, in 13 patients, treatment-related grade 3 or 4 adverse events (AE; 28.3\%) and inflammatory toxicities were reported and were generally higher than expected with ipilimumab alone but remained manageable [23]. In NSCLC, current trials are investigating various combinations of immunomodulatory therapies and systemic therapy including bevacizumab (NCT02039674) as well as nivolumab and bevacizumab maintenance therapy (NCT01454102). There is no doubt that such combinations might also represent a promising concept for $\mathrm{BC}$ patients with a special focus again on the TNBC subtype, although the proof of principle requires further clinical trials.

\section{Targeted Agents and IO}

Moreover, there is a strong rationale regarding the synergism between novel agents in combination with an immunotherapeutic approach. Only a few weeks ago, preliminary data of a first trial combining cyclin-dependent kinase (CDK) inhibitors with CPI were presented at the $\mathrm{BC}$ meeting in San Antonio in December 2017. 28 luminal BC patients pretreated with up to 2 regimens for metastatic disease received abemaciclib together with pembrolizumab. After 16 weeks, a DCR of $75 \%$ was observed (61\% stable disease, $14 \%$ partial response). However, a longer follow-up period is needed to perform a final analysis of the efficacy data.

\section{Side Effects}

One key issue for IO approaches and in particular for combinatorial therapies is whether antitumor effects can be achieved without a corresponding increase in serious toxicities. Clinical studies investigating immunotherapeutic agents as monotherapy have demonstrated an acceptable safety profile in general, particularly compared with conventional cytotoxics. Most immune-related AEs seemed to be reversible and of inflammatory nature. However, safety and efficacy need to be further characterized in larger trials. Combining conventional antitumor agents with immunotherapies usually results in combining and adding toxicities known from the respective drugs as monotherapies. For example, a phase II trial evaluating ipilimumab combined with platinum-based chemotherapy in NSCLC demonstrated similar non-hematologic AEs typically associated with carboplatin and paclitaxel.

PD-1 inhibitors are associated with a specific spectrum of immune-related AEs. This spectrum is different from toxicities known for kinase inhibitors or cytotoxic drugs. Immunologic side effects include - apart from general disorders and administration site conditions - skin, gastrointestinal, pulmonary, endocrine, renal, and hepatic events [24]. For the 6 main events, namely rash, colitis, pneumonitis, endocrinopathies, renal dysfunction (nephritis), and hepatitis, an extensive educational program was agreed between the European Medicines Agency and the drug manufacturer as an additional risk minimization procedure. In the case of pembrolizumab, in addition to the previously listed events, uveitis, pancreatitis, type 1 diabetes mellitus, and myositis have been identified as further key events $[25,26]$.

\section{Skin Toxicity}

Skin events are one of the most commonly occurring side effects in the pivotal clinical studies of the PD-1 inhibitors. These AEs 
include pruritus, rash, dermatitis, erythema, palmoplantar erythrodysesthesia, photosensitivity reaction, toxic epidermal necrolysis, urticaria, and vitiligo. Most of the observed events are immunerelated, with grade 3-4 events reported in less than $1 \%$ of patients treated with nivolumab. For pembrolizumab $2 \mathrm{mg} / \mathrm{kg} \mathrm{q} 3 \mathrm{w}$, immune-related 'severe skin reactions' of grade 3 were seen in $1.5 \%$ of melanoma patients. The median time of onset was in the range of 4-8 weeks for nivolumab and 23 weeks for pembrolizumab. Most patients were treated with local glucocorticoids, while only selected melanoma patients received systemic glucocorticoid treatment. None of the patients in the NSCLC studies received high-dose methylprednisolone or equivalent to resolve skin reactions. Intermittent or permanent discontinuation of the PD-1 inhibitor was rarely necessary [27].

\section{Gastrointestinal Events}

Median time to onset of AEs was in the range of 6 weeks for nivolumab, with a considerably longer median (18 weeks) reported for colitis of any grade for pembrolizumab. Patients can present with complaints of diarrhea (watery or bloody), abdominal pain, fever, anal pain, rectal bleeding, weight loss, and nausea/ vomiting. When immune-related diarrhea is suspected, a stepwise approach depending on its severity is recommended. Grade $1 \mathrm{di}-$ arrhea/colitis can be managed with antidiarrheal medications, oral hydration, and electrolyte supplements. In grade 2 or 3 cases, anti-PD-1 therapy should be withheld, and glucocorticoids (1-2 $\mathrm{mg} / \mathrm{kg} /$ day methylprednisolone equivalent for grade 3, $0.5-1 \mathrm{mg} /$ $\mathrm{kg} /$ day for persistent grade 2 events) should be administered which generally improve symptoms within 1-2 weeks. Upon therapy improvement, anti-PD-1 therapy may be resumed after glucocorticoid taper, which should be carried out over at least 1 month to prevent symptom rebound. In refractory cases, the therapy must be permanently discontinued, and immunosuppressive treatment should be escalated. Infliximab can be considered for treating cases refractory to steroids after 3-5 days [28]. For grade 4 diarrhea or colitis, PD-1 inhibitor therapy must be permanently discontinued, and medical therapy should be given as described above. The potential risk of gastrointestinal perforation should be kept in mind [29].

\section{Pulmonary Toxicity}

The main immune-related respiratory $\mathrm{AE}$ that has occurred with a severity of grade $3-4$ in up to $1.6 \%$ of patients is pneumonitis, representing the most common treatment-related event leading to discontinuation of immunotherapy. The median time of onset of pneumonitis as a select immune AE in melanoma patients was considerably later in the case of pembrolizumab (19 weeks; range 0.3-84 weeks) compared to nivolumab (9 weeks; range 4-26 weeks). An early diagnosis as well as removal from exposure and therapy initiation are essential for the course of the disease, which is treated with immunosuppressive agents, preferably glucocorticoids ( $1 \mathrm{mg} / \mathrm{kg} /$ day methylprednisolone or equivalent in the case of grade $2,2-4 \mathrm{mg} / \mathrm{kg} / \mathrm{day}$ if grade $3-4$ is diagnosed).

\section{Endocrine Events}

Endocrine disorders of any grade associated with anti-PD-1 therapy have been observed in up to $10 \%$ of all treated patients. These endocrine toxicities are generally autoimmune-mediated and mostly affect the thyroid gland resulting in grade 1-2 hypothyroidism (4-8\%) and hyperthyroidism (2-3\%) as well as less frequently in acute thyreoiditis (1\%). Uncommonly, hypophysitis (0.2-1\%), hypopituitarism (0.1-0.2\%), and type 1 diabetes mellitus $(0.1 \%)$ may occur $[25,30]$. Symptoms that may indicate endocrine dysfunction include unusual headaches, extreme tiredness, weight gain or weight loss, changes in mood or behavior such as decreased sex drive, irritability, or forgetfulness, dizziness or fainting, hair loss, feeling cold, constipation, and a deeper pitch of the voice. In addition, the very rare event of adrenal crisis must be considered if dehydration, hypotonia, or even shock symptoms develop.

Autoimmune thyroid disease usually presents in the form of chronic lymphatic thyreoiditis (Hashimoto's disease). The thyroidstimulating hormone count is increased, and fT4 and/or fT3 are below the reference range. Anti-thyroid peroxidase as well as antithyroglobulin antibodies have been reported to become detectable or increase with PD-1 inhibitor therapy. Therefore, routine measurement of thyroid antibodies is recommended [31]. In general, endocrine events - their median time to onset varies from 4 to 18 weeks - are rarely the reason to permanently discontinue PD-1 inhibitor treatment because hormone replacement therapy can be given alongside the anticancer treatment. For patients with grade 3 or grade 4 endocrinopathy that improved to grade 2 or lower with or without hormone replacement, PD-1 inhibitor treatment can be continued after glucocorticoid taper if needed.

However, it is noteworthy that PD-1 inhibitors showed lower rates of drug-related AEs than conventional chemotherapies used as comparators [32]. The frequency of grade 3-4 AEs, not only in disorders susceptible to immune-related select AEs but also in all standard organ classes, was $62 \%$ for any event term. The only exception was grade 3 fatigue reported in $2.4 \%$ of patients. Therefore, due to the highly variable times of onset of immune-related toxicities (some events like pneumonitis or endocrine events often occur late), clinicians should extend the safety monitoring period beyond the drug administration period with regular follow-up visits [33]. Early detection is key to successfully managing these mostly reversible events. Of note, after the occurrence of an immune-related event, anti-PD-1 therapy might be delayed or even stopped in certain situations. Dose reductions are not an adequate measure for CPIs [34]. After resolution of events, or, in some situations, after their attenuation, anti-PD-1 treatment should be restarted whenever possible. As the vast majority of AEs is mild to moderate and reversible following immunosuppressive therapy, re-initiation after recovery represents a therapeutic option for patients [35].

\section{Conclusion}

There is growing evidence that the blockade of PD-1/PD-L1, predominantly as a monotherapy, is very effective in numerous 
solid tumors and also in some hematologic malignancies. In contrast, the activity of anti-PD-(L)1 antibodies as a monotherapy is moderate in metastatic BC. However, these findings have to be interpreted with caution with respect to the small database and the heavily pretreated patients included in the majority of the analyzed trials. Moreover, the role of biomarkers such as PD-L1 expression as a predictor of response to these drugs is still under debate.
Currently, many trials in $\mathrm{BC}$ focus on combination regimens with cytotoxics in TNBC subtypes as the target population. Based on these considerations, patients with $\mathrm{BC}$ who meet the criteria for the use of CPI should be treated preferentially in clinical trials.

\section{Disclosure Statement}

JB served on advisory boards for Novartis and Pfizer and received honoraria from Astra Zeneca.

\section{References}

1 Yao S, Zhu Y, Chen L: Advances in targeting cell surface sigalling molecules for immune modulation. Nat Ref Drug Discov 2013;12:130-146.

2 Brunet JF, Denizot F, Lucianni MF, et al: A new member of the immunoglobulin superfamily - CTLA -4 . Nature 1987;328:267-270

3 Chemnitz JM, Parry RV, Nichols KE, et al: SHP-1 and SHP-2 associate with immunoreceptor tyrosine-based switch motif of programmed death 1 upon primary human $\mathrm{T}$ cell stimulation, but only receptor ligation prevents T cell activation. J Immunol 2004;173:945954.

4 Pardoll DM: The blockade of immune checkpoints in cancer immunotherapy. Nat Rev Cancer 2012;12:252264.

5 Sage PT, Francisco LM, Carman CV, Shape AH: The receptor PD-1 controls follicular regulatory $\mathrm{T}$ cells in the lymph nodes and blood. Nat Immunol 2013;14: 152-181.

6 Hodi FS, O’Day SJ, McDermott DF, et al: Improved survival with ipilimumab in patients with metastatic melanoma. N Engl J Med 2010;363:711-723.

7 Ishida Y, Agata Y, Shibahara K, Honjo T: Induced expression of PD-1, a novel member of the immunoglobulin gene superfamily, upon programmed cell death. EMBO J 1992;11:3887-3895

8 Freeman GJ, Long AJ, Iwai Y, et al: Engagement of the PD-1 immunoinhibitory receptor by a novel B7 family member leads to negative regulation of lymphocyte activation J Exp Med 2000;192:1027-1034.

9 Ahmadyadeh M, Johnson LA, Heemskerk B, et al: Tumor antigen-specific CD8 $\mathrm{T}$ cells infiltration the tumor express high levels of PD-1 and are functionally impaired. Blood 2009;114:1537-1544.

10 Ghebeh H, Mohammed S, Al-Omair A, et al: The $\mathrm{B} /-\mathrm{H} 1$ (PD-L1) T lymphocyte-inhibitory molecule is expressed in breast cancer patient with infiltrating ductal carcinoma: correlation with important high risk prognostic factors. Neoplasia 2006;8:190-198.

11 Ali HR, Glont SE, Blows FM, et al: PD-L1 protein expression in breast cancer is rare, enriched in basal-like tumours and associated with infiltrating lymphocytes. Ann Oncol 2015;26:1488-1493.
12 Anda R, Chow LQ, Dees EC, et al: A phase Ib study of pembrolizumab in patients with advanced triple negative breast cancer. Cancer Res 2015;75:abstr S1-09.

13 Emens LA, Braiteh FB, Cassier P, et al: Inhibition of PD-L1by MPDL3280A leads to clinical activity in patients with metastatic triple negative breast cancer. Cancer Res 2015;75:abstr PD1-6.

14 Adams S, Schmid P, Rugo HS, et al: Phase 2 study of pembrolizumab monotherapy for previously treated metastatic triple-negative breast cancer (mTNBC): KEYNOTE-086 cohort A. J Clin Oncol 2017;35:1008.

15 Kroemer G, Galluzzi L, Kepp O, et al: Immunogenic cell death in cancer therapy. Ann Rev Immunol 2013; 31:51-72.

16 Voll RE, Herrmann M, Roth EA, et al: Immunosupressive effects of apoptotic cells. Nature 1997;390:350351 .

17 Casares N, Pequignot MO, Tesniere A, et al: Caspasedependent immunogenicity of doxorubicin-induced tumor cell death. J Exp Med 2005;202:1691-1701.

18 Apetoh L, Ghiringhelli F, Tesniere A, et al: Toll-like receptor 4-dependent contribution of the immune system to anticancer chemotherapy and radiotherapy. Nat Med 2007;13:1050-1059.

19 Krysko DV, Garg AD, Kaczmarek A, et al: Immunogenic cell death and DAMPs in cancer therapy. Nat Rev Cancer 2012;12:860-875.

20 Hannani D, Sistigu A, Kepp O, et al: Prerequisites for the antitumor vaccine-like effect of chemotherapy and radiotherapy. Cancer J 2011;17:351-358.

21 Carmeliet P, Jain RK: Molecular mechanisms and clinical applications of angiogenesis. Nature 2011;473: 298-307.

22 Gabrilovich DI, Isida T, Nadaf S, et al: Antibodies to vascular endothelial growth factor enhance the efficacy of cancer immunotherapy by improving endogenous dendritic cell function. Clin Cancer Res 1999;5:29632970.

23 Hodi FS, Lawrence D, Lezcano C, et al: Bevacizumab plus ipilimumab in patients with metastatic melanoma. Cancer Immunol Res 2014;2:632-642.
24 Weber JS, Antonia SJ, Topalian SL, et al: Safety profile of nivolumab in patients with advanced melanoma - a pooled analysis. J Clin Oncol 2015;33(suppl):abstr 9018

25 Gaudy C, Clevy C, Monestier S, et al: Anti-PD1 pembrolizumab can induce exceptional fulminant type 1 diabetes. Diabetes Care 2015;38:e182-183.

26 Manusow JS, Khoja L, Pesin N, et al: Retinal vasculitis and ocular vitreous metastasis following complete response to PD-1 inhibition in a patient with melanoma. J Immunother Cancer 2014;2:41

27 Abdel-Rahman O, ElHalawani H, Fouad M: Risk of cutaneous toxicities in patients with solid tumors treated with immune checkpoint inhibitors: a metaanalysis. Future Oncol 2015;11:2471-2484

28 Pagès C, Gornet JM, Monsel G, et al: Ipilimumab-induced acute severe colitis treated by infliximab. Melanoma Res 2013;23:227-230.

29 Cheng R, Cooper A, Kench J, et al: Ipilimumab-induced toxicities and the gastroenterologist. J Gastroenterol Hepatol 2015;30:657-666.

30 Albarel F, Gaudy C, Castinetti F, et al: Long term follow up of ipilimumab-induced hypophysitis, a common AE of the anti-CTLA-4 antibody in melanoma Eur J Endocrinol 2015; 172:195-204

31 Ryder M, Callahan M, Postow MA, et al: Endocrinerelated AEs following ipilimumab in patients with advanced melanoma - a comprehensive retrospective review from a single institution. Endocr Relat Cancer 2014;21:371-381.

32 Brahmer J, Reckamp KL, Baas P, et al: Nivolumab vs. docetaxel in advanced NSCLC. N Engl J Med 2015;373: 123-135.

33 Weber JS, Kähler KC, Hauschild A: Management of immune-related adverse events and kinetics of response with ipilimumab. J Clin Oncol 2012;30:2691-2697.

34 Harrid O, Robert C, Daud, et al: Safety and tumor responses with lambrolizumab in melanoma. N Engl J Med 2013;369:134-144

35 Topalian SL, Hodi FS, Brahmer JR, et al: Safety, activity and immune correlates of anti-PD-1 antibody in cancer. N Engl J Med 2012;366:2443-2454. 